УДК 616-056.25-055.2-036.868:613.2

( Н.И. Храмцова, Т.М. Лебедева, Ю.Ю. Заякин, С.А. Плаксин, Т.В. Шагова

ГБОУ ВПО «Пермский государственный медицинский университет имени академика Е.A. Вагнера» Минздрава России

Г. Пермь, Россия

\author{
ВЗАИМОСВЯЗЬ КАЧЕСТВА ЖИЗНИ, ОБУСЛОВЛЕННОГО ОТНОШЕНИЕМ \\ К СОБСТВЕННОЙ ВНЕШНОСТИ, С ОСОБЕННОСТЯМИ ПИЩЕВОГО ПОВЕДЕ- \\ НИЯ У ЖЕНЩИН С ЛОКАЛЬНОЙ ЛИПОДИСТРОФИЕЙ
}

\begin{abstract}
Аннотация. Проанализированы шкала пищевого поведения и общий балл опросника BIQLI у 15 женщин с локальной липодистрофией и 35 здоровых женщин. Определена взаимосвязь между особенностями пищевого поведения и качеством жизни, обусловленного отношением к собственной внешности $(r=0,5, r=0,7 ; p=0,0004)$. У пациенток с липодистрофиями отношение к своей внешности положительно влияет на возможность контролировать пищевое поведение и вес. Уровень контроля над характером питания, связанный с отношением к своей внешности, сопоставим $0,7 \pm 1,5$ и $0,7 \pm 2,0$ баллов. Уровень контроля над весом, связанный с отношением к собственной внешности, несколько выше в группе пациенток с липодистрофиями - 0,9 $\pm 1,6$ и $0,3 \pm 1,6$ баллов в группе сравнения. Уровень качества жизни, обусловленный отношением к собственной внешности, выше у женщин с липодистрофией $-28,1 \pm 13,6$ и $21,2 \pm 16,0$ в группе сравнения $(\mathrm{p}=0,1)$. В комплексе диагностических методов у пациентов с локальными липодистрофиями целесообразно учитывать особенности отношения к собственной внешности, обусловленные возможными расстройствами пищевого поведения, которые служат противопоказанием к оперативному лечению.
\end{abstract}

Ключевые слова: опросник, BIQLI, пищевое поведение, липосакция, качество жизни.

(c) N.I. Khramtsova, T.M. Lebedeva, Yu.Yu. Zayakin, S.A. Plaksin, T.V. Shagova

Perm State Medical University named after E.A. Wagner

Perm, Russia

\title{
INTERRELATION OF LIFE QUALITY DETERMINED BY THE ATTITUDE TO OWN BODY IMAGE WITH FEATURES OF EATING BEHAVIOR IN WOMEN WITH LOCAL LIPODYSTROPHY
}

Abstract. Eating behavior scale and total score of BIQLI questionnaire were analyzed in 15 women with local lipodystrophy, and 35 healthy women. The correlation between eating behavior and the quality of life determined by own body image was found $(r=0,5, r=0,7 ; p=0,0004)$. In patients with lipodystrophy the body image has a positive effect on the ability to control eating behavior and weight. The body image related to eating behavior was comparable $-0,7 \pm 1,5$ and $0,7 \pm 2,0$. The body image related to weight control was slightly higher in the lipodystrophy group - $0,9 \pm 1,6$ and $0,3 \pm 1,6$ points in the comparison group. The level of body image quality of life was higher in women with lipodystrophy $-28,1 \pm 13,6$ and $21,2 \pm 16,0$ in the control group $(p=0.1)$. Diagnostic methods in patients with lipodystrophy should include the body image assessment and eating disorders exclusion.

Keywords: questionnaire, BIQLI, eating behavior, liposuction, quality of life.

Ведение. Отношение к собственной внешности отражается на многих не только объективных (социальное и персональное функционирование), но и субъективных (эмоциональный фон, душевное самочувствие, самооценка, 
состояние счастья) аспектах жизни человека и, в конечном итоге, определяет удовлетворенность личности качеством своей жизни в целом $[1,3]$. Будучи многомерным по происхождению, отношение к своей внешности во многом связано с пищевым поведением и его аномалиями, непосредственно влияющими как на физические параметры тела, так и на психологическое и социальное функционирование личности. Расстройства пищевого поведения включают в себя не только психиатрические заболевания, такие как анорексию и булимию, но и субклинические формы, в частности, ощущение себя «толстым» и стремление в связи с этим «во что бы то ни стало снизить свой вес» [2], меняя привычные пищевые стереотипы либо прибегая к хирургическим методам коррекции. Однако в научной литературе публикации по данной проблематике носят ограниченный характер и охватывают в основном лишь самые общие вопросы. Кроме того, недостаточно изучена взаимосвязь качества жизни и особенностей пищевого поведения у группы лиц, прибегающих к хирургической коррекции контура тела. Таким образом, анализ отношения к собственной внешности во взаимосвязи с характером пищевого поведения представляет глубокий теоретический и практический интерес.

Цель исследования заключалась в изучении взаимосвязи качества жизни, обусловленного отношением к собственной внешности, с особенностями пищевого поведения у женщин с локальной липодистрофией.

Материалы и методы. В рамках настоящего исследования были проанализированы данные 15 женщин с локальной липодистрофией, имеющих медицинские показания к липосакции. В группу сравнения вошли 35 практически здоровых женщин. Изученные группы сопоставимы по основным демографическим и социальным характеристикам. Средний возраст равнялся $37,6 \pm 8,2$ и $34,9 \pm 7,9$ лет $(p=0,2)$ соответственно. Масса тела и индекс массы

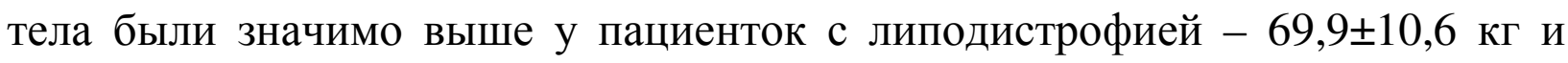
$58,9 \pm 7,6$ кг $(\mathrm{p}=0,007) ; 25,7 \pm 4,8$ и $22,0 \pm 2,1$ кг/ $\mathrm{M}^{2}(\mathrm{p}=0,01)$ соответственно. 
Критерии включения: женщины с локальной липодистрофией и нормальной или избыточной массой тела (индекс массы тела от 18,5 до 29,9

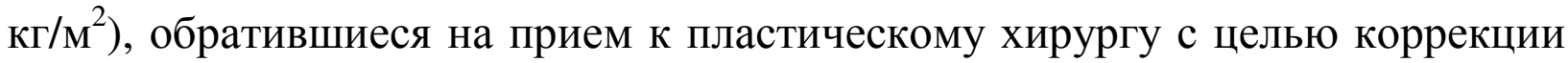
контура фигуры, имеющие уровень образования не ниже среднего специального и давшие информированное добровольное согласие на участие в научном клиническом и экспериментально-психологическом исследовании.

Критерии исключения: психические расстройства, тяжелые соматические заболевания, профессиональное занятие спортом.

Для достижения поставленной цели были использованы следующие методы: клинико-анамнестический метод для выявления социальных и демографических параметров, мотивации для обращения к пластическому хирургу, прогнозирования ожидаемых результатов; антропометрический метод для оценки объективных физических параметров; опросник BIQLI, включающий в себя общий балл и шкалу пищевого поведения для изучения уровня качества жизни, связанного с отношением к собственной внешности, а также оценки влияния собственной внешности на модель пищевого поведения [2]. Для установления степени значимости использованы коэффициенты корреляции Спирмена, критерии Вальда-Волфовитца и Фишера. Статистическая значимость теста принималась при величине ошибки $\mathrm{p}<0,05$. Данные обработаны с помощью пакетов Microsoft Excel 2007 и Statistica 8.0.

\section{Результаты и их обсуждение.}

Анализ особенностей пищевого поведения в зависимости от отношения к собственной внешности представлен на рисунке 1. 


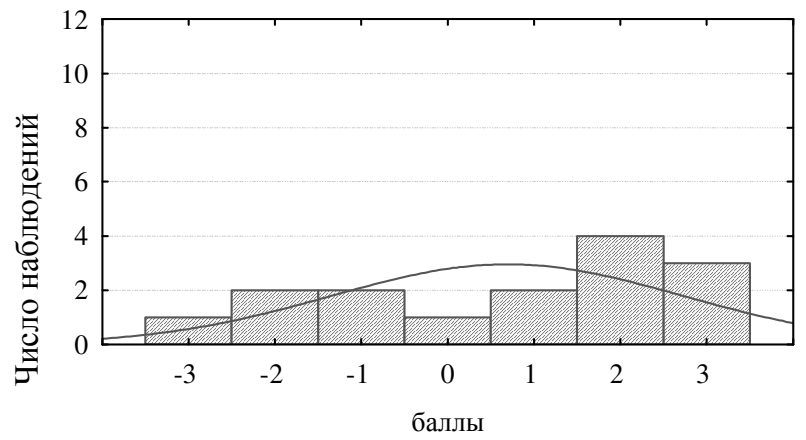

пациенты с липодистрофией

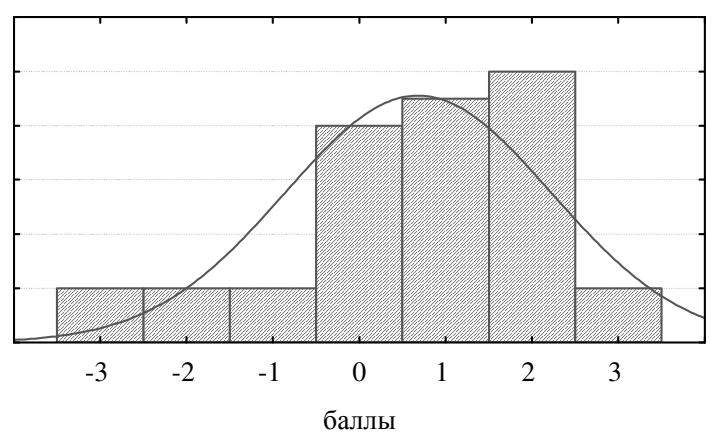

контрольная группа

Рис. 1. Гистограмма влияния отношения к собственной внешности на «возможность контролировать, что и сколько я ем»

Средний балл по вопросу «Возможность контролировать, что и сколько я ем» в группе пациенток с липодистрофией сопоставим с таковым в группе сравнения: $0,7 \pm 2,0$ и $0,7 \pm 1,5$ соответственно. При анализе распределения ответов видно, что у пациенток с липодистрофией спектр влияния внешности на способность контролировать количество и качество принимаемой пищи распределен почти равномерно (p=0,14). У 9 (60\%) женщин с липодистрофией определяется положительное влияние внешности на этот аспект пищевого поведения, отрицательное влияние внешности - у 5 (33\%).

На гистограмме распределения ответов в группе сравнения 21 (60\%) приходится на положительное влияние внешности, отрицательное влияние у 6 (17\%); p=0,0002. В группе сравнения отсутствие влияния внешности на особенности пищевого поведения отмечается у 8 (27\%) женщин, в то время как в опытной группе - лишь у 1 (6,7\%).

Различие между группами статистически не значимо ( $\mathrm{p}=0,86)$, однако при анализе гистограмм наблюдается тенденция к более высоким значениям в группе сравнения. То есть практически здоровые женщины с меньшим индексом массы тела отмечают более положительное влияние своей внешности на возможность контролировать пищевое поведение. 
Анализ способности контролировать свой вес в зависимости от отношения к собственной внешности представлен на рисунке 2.

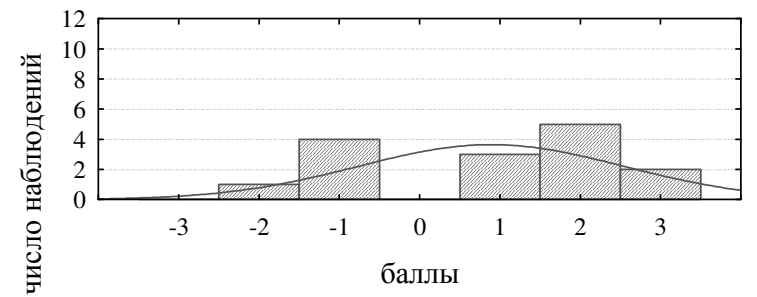

пациенты с липодистрофией

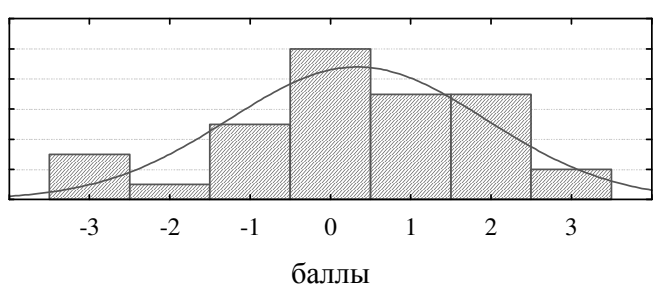

контрольная группа

Рис. 2. Гистограмма влияния отношения к собственной внешности на «способность контролировать свой вес»

Средний балл по влиянию внешности на способность контролировать свой вес в группе женщин с локальной липодистрофией больше, чем в группе сравнения $-0,9 \pm 1,6$ и $0,3 \pm 1,6$ баллов соответственно $(\mathrm{p}=0,39)$.

При анализе гистограмм распределения ответов, касающихся влияния внешности на способность контролировать свой вес, обращает на себя внимание отсутствие «нулевых» ответов для группы лиц с липодистрофиями, то есть для каждой из них внешность имеет влияние на этот параметр.

Для 10 (67\%) женщин с локальной липодистрофией внешность имеет положительное влияние на способность контролировать свой вес, для 5 (33\%) опрошенных - легкий или умеренный отрицательный эффект; $\mathrm{p}=0,07$.

Из группы здоровых лиц для 16 (46\%) респондентов внешность имеет положительное влияние на способность контролировать свой вес, не имеет влияния у 10 (29\%), отрицательное - у 9 (25\%); $\mathrm{p}=0,07$.

Таким образом, в обеих группах внешность имеет положительное влияние на возможность контролировать свой вес с тенденцией к более выраженному влиянию в группе пациенток с липодистрофией.

Анализ уровня качества жизни, обусловленного отношением к собственной внешности, представлен на рисунке 3. 


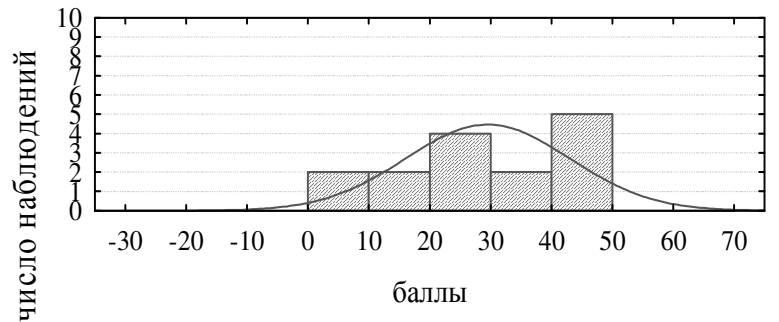

пациенты с липодистрофией

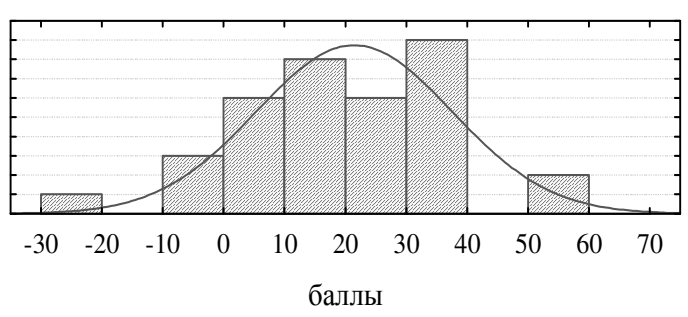

контрольная группа

Рис. 3. Гистограмма распределения уровня качества жизни, обусловленного отношением к собственной внешности

Общий уровень качества жизни, связанного с отношением к собственной внешности, в группе женщин с липодистрофией был выше, чем в группе

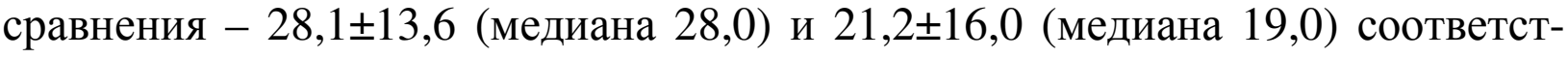
венно $(\mathrm{p}=0,1)$. В этой группе отсутствуют отрицательные значения.

В группе сравнения у 3 (9\%) женщин общий уровень качества жизни был отрицательным, у 1 (3\%) - равнялся нулю. В остальных случаях - 31 (88\%) - уровень качества жизни был положительным.

Выявлено, что на суммарный индекс качества жизни повлияли баллы по ответам оба вопроса шкалы пищевого поведения: способность контролировать характер питания $(\mathrm{R}=0,5 ; \mathrm{p}=0,0004)$ и способность контролировать свой вес $(\mathrm{R}=0,7 ; \mathrm{p}=0,0001)$.

Выводы. 1. Определена значимая взаимосвязь между особенностями пищевого поведения и качеством жизни, обусловленного отношением к собственной внешности. Тенденции, не имеющие достоверности, требуют более углубленного анализа и увеличения объема выборок.

2. У пациенток с локальными липодистрофиями отношение к своей внешности положительно влияет на возможность контролировать пищевое поведение и вес. Уровень контроля над характером питания, связанный с отношением к своей внешности, имеет тенденцию к более высоким показателям в группе здоровых женщин. Уровень контроля над весом, связанный с отношением к собственной внешности, выше в группе пациенток с локальными липодистрофиями. 
3. С учетом сопоставимой либо более низкой способности контроля за пищевым поведением и более высокой способностью контролировать свой вес у женщин с липодистрофией, можно предположить, что контроль веса в этой группе женщин осуществляется не только за счет диеты, но другими способами.

4. Уровень качества жизни, обусловленный отношением к собственной внешности, выше у женщин с липодистрофией. Несмотря на наличие корреляции между показателями пищевого поведения с уровнем качества жизни, на наш взгляд, на итоговый показатель повлияли и другие аспекты психологического и социального функционирования.

Практические рекомендации: В комплексе диагностических методов у пациентов с локальными липодистрофиями целесообразно учитывать особенности отношения к собственной внешности, обусловленные возможными расстройствами пищевого поведения, которые можно рассматривать как одно из противопоказаний для оперативной коррекции контура тела.

\section{Список литературы:}

1. Cash T.F. The impact of body image experiences: development of the body image quality of life inventory / Cash T.F., Fleming E.C. // Int J Eat Disord. 2002 May;31(4):455-60.

2. Cook B. Exercise in Eating Disorders Treatment: Systematic Review and Proposal of Guidelines / Cook B., Wonderlich S.A., Mitchell J., Thompson R., Sherman R., McCallum K. // Med Sci Sports Exerc. 2016, Feb, 24, [Epub ahead of print]..

3. Jáuregui Lobera I., Bolaños Ríos P. Body image quality of life in eating disorders / Patient Prefer Adherence. 2011 Mar 3;5:109-16.

\section{References}

1. Cash T.F., Fleming E.C. The impact of body image experiences: development of the body image quality of life. Int J Eat Disord., 2002, May, no. 31(4), pp. 455-60.

2. Cook B., Wonderlich S.A., Mitchell J., Thompson R., Sherman R., McCallum K. Exercise in Eating Disorders Treatment: Systematic Review and Proposal of Guidelines. Med Sci Sports Exerc., 2016, February, no. 24, [Epub ahead of print]. 
3. Jáuregui Lobera I., Bolaños Ríos P. Body image quality of life in eating disorders. Patient Prefer Adherence,.2011, March, no. 3(5), pp. 109-16.

Храмцова Наталья Игоревна - к.м.н., ассистент кафедры госпитальной хирургии и кафедры общественного здоровья и здравоохранения ГБОУ ВПО ПГМУ им. академика Е.А.Вагнера Минздрава России. E-mail: renelve@ mail.ru. Тел. (+7342) 233-23-36.

Лебедева Татьяна Михайловна - д.м.н., профессор кафедры общественного здоровья и здравоохранения ГБОУ ВПО ПГМУ им. академика Е.А.Вагнера Минздрава России. Еmail: super.oziz@yandex.ru. Тел. (+7342) 233-23-36.

Заякин Юрий Юрьевич - к.м.н., доцент кафедры психиатрии наркологии и медицинской психологии ГБОУ ВПО ПГМУ им. академика Е.А.Вагнера Минздрава России. Тел. (+7342) 236-46-51. E-mail: zayakin@inbox.ru.

Плаксин Сергей Александрович - д.м.н., профессор кафедры хирургии ФДПО ГБОУ ВПО ПГМУ им. академика Е.А.Вагнера Минздрава России. E-mail: splaksin@mail.ru. Тел. (+7342) 239-29-72.

Шагова Татьяна Васильевна - к.м.н., доцент кафедры общественного здоровья и здравоохранения. E-mail: super.oziz@ yandex.ru Тел. (+7342) 233-23-36.

ГБОУ ВПО «Пермский государственный университет им. академика Е.А.Вагнера Минздрава России» Россия, 614990 Пермь, ул.Петропавловская, 26.

Khramtsova Natalya Igorevna - Candidate of Medical Science, teaching assistant of the department of hospital surgery, the department of public health and healthcare, Perm State Medical University named after E.A. Wagner, phone: (+7342) 233-23-36, e-mail: renelve@ mail.ru.

Lebedeva Tatyana Mikhaylovna - Doctor of Medical Science, professor of the department of public health and healthcare, Perm State Medical University named after E.A. Wagner, phone: (+7342) 233-23-36, e-mail: super.oziz@ yandex.ru.

Zayakin Yuriy Yuryevich - Candidate of Medical Science, associate professor of the department of narcology and medical psychology, Perm State Medical University named after E.A. Wagner, phone: (+7342) 236-46-51, e-mail: zayakin@inbox.ru

Plaksin Sergey Aleksandrovich - Doctor of Medical Science, professor of the department of surgery of the faculty of advanced doctors` training, Perm State Medical University named after E.A. Wagner, phone: (+7342) 239-29-72, e-mail: splaksin@mail.ru

Shagova Tatyana Vasilyevna - Candidate of Medical Science, associate professor of the department of public health and healthcare, Perm State Medical University named after E.A. Wagner, phone: (+7342) 233-23-36, e-mail: super.oziz@yandex.ru 
State Budgetary Educational Institution of Higher Professional Training "Perm State Medical University named after E.A. Wagner" of the RF Ministry of Health, 26, Petropavlovskaya str., 614990, Perm, Russia, 